\title{
Efficient slow-light coupling in a photonic crystal waveguide without transition region
}

\author{
T. P. White, ${ }^{1, *}$ L. C. Botten, ${ }^{2}$ C. Martijn de Sterke, ${ }^{3}$ K. B. Dossou, ${ }^{2}$ and R. C. McPhedran ${ }^{3}$ \\ ${ }^{1}$ School of Physics and Astronomy, University of St Andrews, St Andrews, Fife, KY169SS, UK \\ ${ }^{2}$ CUDOS, Department of Mathematical Sciences, University of Technology, Sydney, \\ New South Wales 2007, Australia \\ ${ }^{3}$ CUDOS, School of Physics, University of Sydney, New South Wales 2006, Australia \\ *Corresponding author: tom.white@st-andrews.ac.uk
}

Received July 16, 2008; revised September 24, 2008; accepted September 25, 2008; posted October 16, 2008 (Doc. ID 98859); published November 12, 2008

\begin{abstract}
We consider the coupling into a slow mode that appears near an inflection point in the band structure of a photonic crystal waveguide. Remarkably, the coupling into this slow mode, which has a group index $n_{g}$ $>1000$, can be essentially perfect without any transition region. We show that this efficient coupling occurs thanks to an evanescent mode in the slow medium, which has appreciable amplitude and helps satisfy the boundary conditions but does not transport any energy. (C) 2008 Optical Society of America

OCIS codes: $130.5296,350.4238$.
\end{abstract}

Slow light has been observed in many geometries, including photonic crystals (PCs) [1]. PCs are particularly suited for this, since the dispersion relation of PC waveguide modes can be controlled and designed with the high accuracy required to achieve very low group velocities $v_{g}$. A key practical challenge with slow light in PCs is the difficulty of coupling light into these waveguides, since the field matches poorly to that of other modes, i.e., modes that are not slow. A number of strategies to deal with this coupling problem have been reported. The first is the use of an adiabatic taper [2], but these tend to be long and lack a systematic design procedure. The second is the use of a uniform matching region [3-5]. Though this works well, it obviously requires the inclusion of an additional, finite region. Here, we discuss a third approach that does not require any matching region. We show that efficient coupling into a slow mode can be mediated by an evanescent mode that does not carry energy but helps match the slow mode's field to that of other modes. Though evanescent modes have been identified to play a role in coupling to slow PC waveguide modes [5], the mechanism was not studied in detail.

Our geometry is illustrated in Fig. 1; light is incident through PC1, a silicon PC with a waveguide and with $a / d=0.3$, where $a$ is the radius of the air holes and $d$ is its period. The input waveguide's properties have been adjusted by changing the radii of the holes two rows from the waveguide to $a_{1}=0.38 d$. The slowlight waveguide in PC2 is identical, except that its properties have been adjusted by taking $a_{2}=0.404 \mathrm{~d}$.

At frequency $d / \lambda=0.2662$, where $\lambda$ is the wavelength, the waveguides in PC1 and PC2 each support a single propagating mode with group indices $n_{g}$ $=7.6$ and $n_{g}=1067$, respectively. These calculations, and all those below, model the PC as two dimensional by taking the effective index of the silicon background to be 2.86. Our computational method [6] generates Fresnel interface coefficients very accurately from a complete Bloch-mode basis that includes modes that are propagating and evanescent in the di- rection of the waveguide. Because of the orthogonality of the Bloch modes [6], we may solve the interface field matching problem in a least-squares sense and, in doing so, identify a minimal set of modes that efficiently solves the problem to given accuracy. Without precautions, the transmittance from PC1 into PC2 is found to exceed $T=99.4 \%$. This is remarkable, since coupling into a slow mode tends to be poor. We now discuss why the transmission might be expected to be low, and then explain why it is almost perfect in the structure considered here.

We first consider the relevant part of the band structures of PC1 and PC2 (solid curves in Fig. 2). PC2 has been designed to have an inflection point where the group velocity $\mathrm{d} \omega / \mathrm{d} k$ becomes very small. High transmission into slow modes close to such an inflection point was earlier noted by Ballato et al. [7] in a one-dimensional geometry. The dashed curves show some of the complex bands, i.e., solutions to Maxwell's equations with complex $\mathbf{k}$ at real frequencies. These solutions, usually ignored, grow or decay exponentially, with $|\operatorname{Im} k|$ indicating the decay rate [at the frequency of operation $\operatorname{Im}(k) d=0.047$ in PC2]. The figures show only the modes with $|\operatorname{Im}(k)| d$ $<0.51$, i.e., modes that are weakly evanescent. Since the dielectric function is real, complex bands occur in complex conjugate pairs, and so each dashed curve represents a pair of evanescent modes.

A thought experiment shows that the presence of

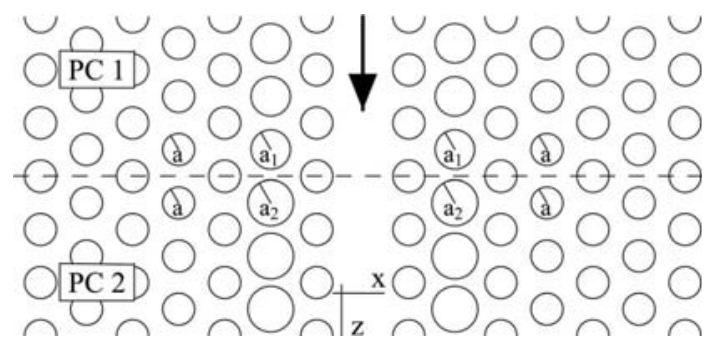

Fig. 1. Schematic of the PC geometry. The light is coupled from PC1 into PC2, which supports a slow mode. 

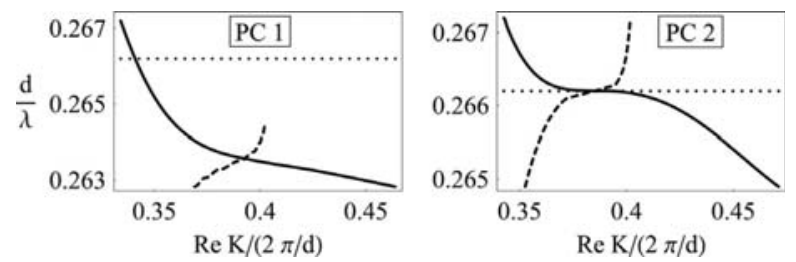

Fig. 2. Part of the band structure of PC1 and PC2 for TE polarization. Propagating modes are indicated by solid curves, evanescent modes with $|\operatorname{Im}(k)| d<0.51$ by dashed curves. The dotted line indicates the inflection point frequency.

the weakly evanescent mode in PC2 is inevitable; starting from PC2, if we apply a suitable small perturbation, for example a change in the hole size or period, or the material's refractive index, then the degeneracy of the zero can be lifted, changing the band to have one local minimum (at frequency $\omega_{\min }$ ) and one maximum (at $\omega_{\max }>\omega_{\min }$ ). There are now thus frequencies $\omega_{\min }<\omega<\omega_{\max }$ with three real solutions for $k$, whereas in the original structure there is only a single real $k$ for each frequency. When the perturbation is removed, two of the three real solutions must become evanescent-since the perturbation is small, $|\operatorname{Im}(k)|$ of these modes must be small too, so they are weakly evanescent. PC1 has no weakly evanescent mode at $d / \lambda=0.2662$, since no mode becomes propagating upon applying a small perturbation.

The reason that coupling into a slow mode tends to be inefficient can be gleaned from the relation $S$ $=U v_{g}$, where $S$ is the power flow and $U$ is the integrated energy density. It shows that for a given energy flow $S$, the energy density for a slow mode is much larger than for other modes. Since field components need to be continuous at an interface, this usually can occur only when the power in the slow mode is low. A more detailed argument uses the Bloch modes and their orthogonality properties. Here, we consider the one deriving from energy conservation. In the next paragraphs we demonstrate that though two modes may be similar, they can nevertheless be orthogonal. Readers not interested in the detailed argument can continue at the paragraph starting with "With this knowledge...."

The Bloch modes are solutions of the eigenvalue equation $\tilde{\mathcal{T f}}=\mu \tilde{f}$, with $\mathcal{T}$ the transfer matrix of a period layer in the structure, $\mu$ associated with the Bloch factor, and $\widetilde{f}$ given by $\tilde{f}^{T}=\left[\tilde{f}_{+}^{T} \tilde{f}_{-}^{T}\right]$ where $\widetilde{f}_{ \pm}$represent the forward- and backward-propagating planewave components [6]. For a propagating mode, $\mu$ $=\exp (i k d)$, where $d$ is the layer period, so $\mu$ lies on the unit circle since the mode amplitude is constant. For evanescent modes, $|\mu| \neq 1$, with $|\mu|$ indicating the ratio of mode amplitudes in consecutive periods. For TE polarization, the case of interest here, the transverse component of the magnetic field on an interface line can be expressed as $H_{z}=f_{+}(x)+f_{-}(x)$, in which $f_{ \pm}(x)=\int \widetilde{f}_{ \pm}(\alpha) \exp (i \alpha x) \mathrm{d} \alpha$, while the transverse component of electric field is $E_{x} / Z=\left(\gamma f_{+}\right)(x)-\left(\gamma f_{-}\right)(x)$, where $Z$ denotes the impedance of the background medium and the $\left(\gamma f_{ \pm}\right)$are defined in an analogous manner as $\left(\gamma f_{ \pm}(x)\right)=\int \sqrt{k^{2}-\alpha^{2}} f_{ \pm}(\alpha) \exp (i \alpha x) \mathrm{d} \alpha$, where $\sqrt{k^{2}-\alpha^{2}}$ is a set of direction cosines.

For hexagonal lattices the Bloch modes at frequency $\omega$ obey the orthogonality relationship

$$
\begin{aligned}
\frac{1}{Z} \int \hat{z} \cdot\left(E_{t}^{m} \times H_{t}^{l *}\right) \mathrm{d} x \\
\quad=\int\left[f_{+}^{l}(x)+f_{-}^{l}(x)\right] *\left[\left(\gamma f_{+}^{m}\right)(x)-\left(\gamma f_{-}^{m}\right)(x)\right] \mathrm{d} x \\
\quad= \begin{cases}\delta_{l m}^{p} & \text { for propagating states } l, m \\
\pm i \delta_{l m}^{e} & \text { for evanescent states } l, m \\
0 & \text { otherwise }\end{cases}
\end{aligned}
$$

for all $l$ and $m$, where $t$ refers to the transverse field component. In Eq. (1) $\delta_{l m}^{p}=1$ if $\mu_{l}=\mu_{m}$ and 0 otherwise, and $\delta_{l m}^{e}=1$ if $\mu_{l}=\mu_{m}^{*}$ and 0 otherwise, respectively, for propagating and evanescent modes. Orthogonality relations [Eq. (1)] take this form because energy may be carried both by propagating states and by evanescent states through tunneling. Though relations like Eq. (1) are familiar in waveguide theory [8], they are not often used in PCs, where one usually exploits the orthogonality at fixed $\mathbf{k}$.

The dots in Fig. 3 show the normalized magnetic field of the slowly propagating mode in PC2, while the solid curve shows that of the scaled weakly evanescent mode that decays away from the interface. Evidently these modes' magnetic fields are very similar, even though they are orthogonal. The reason why two orthogonal modes can nonetheless be very similar can be gleaned from the observation that whereas $\int \hat{z} \cdot\left(E_{t}^{m} \times H_{t}^{l *}\right) \mathrm{d} x / Z$ enters the orthogonality relation (1), $\int \hat{z} \cdot\left|\left(E_{t}^{m} \times H_{t}^{l *}\right)\right| \mathrm{d} x / Z \approx 900$ for the slow mode in PC2. This shows that the field of this mode is large and that the normalization can come about only from many sign changes of the integrand. Therefore small changes in the integrand can change the right-hand side from 0 to 1 or to $i$. That two orthogonal modes can yet be similar is perhaps not surprising, since they are solutions to the Helmholtz equation with the same refractive index, the same frequency, and very similar $\mu$. Though Fig. 3 shows magnetic fields, the same is true for the electric fields.

With this knowledge, we now turn to the coupling problem outlined in Fig. 1: PC2 has a slow mode and
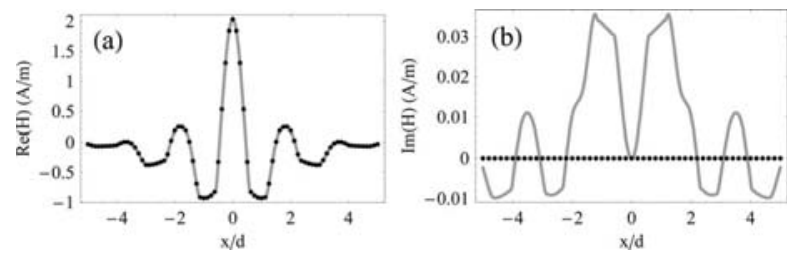

Fig. 3. Normalized magnetic fields of the slow (dots) and the weakly evanescent (solid curves) modes in PC2 (cross section at the top interface), showing (a) the real part and (b) the imaginary part. The slow mode can be scaled to be real, while the normalized evanescent mode has been divided by 0.706 such that its maximum amplitude is real and equals that of the slow mode. 
a weakly evanescent mode that decays away from the interface [9]. Even though these two modes are orthogonal, they have very similar field structures. In contrast PC1 supports no slow mode, and all its nonpropagating modes are strongly evanescent. Since the slow mode and the evanescent mode have very similar fields, the superposition $f_{s}-f_{e} / 0.706$, where $f_{s, e}$ represent the field profiles of the slow and evanescent modes respectively, has a low field strength, and it therefore can match well to the single propagating mode in PC1. Away from the interface the evanescent mode decays and so it cancels the propagating mode's field less and less, leading to an increase of the net field strength. This continues until the evanescent mode decays completely, at which point the net field strength saturates, consistent with Fig. 4 . The periodicity of the rapidly varying field component indicates that the $k$ of the slow mode and $\operatorname{Re}(k)$ of the evanescent mode have essentially the same value (c.f., Fig. 2). Note further that the saturation distance of roughly 40 periods is consistent with the value $|\mu|=\exp (-0.047)=0.954$ of the evanescent mode. The inset in Fig. 4 shows the transmittance and the group velocity versus frequency. Remarkably, the transmission is high over an extended range of low group velocities.

One way to confirm the correctness of the argument above is by calculating the transmissivity $T$ into the slow mode using different numbers of modes. We can do so since we use a least-squares modal method, which exploits modal orthogonality, for our numerical calculations. As mentioned, a full calculation, i.e., a calculation including all relevant modes, gives $T=99.4 \%$. In contrast, including only the propagating mode in $\mathrm{PC} 2$ and all modes in $\mathrm{PC} 1$ gives $T$ $=4.9 \%$. Thus the evanescent modes in PC2 play a key role in achieving the high transmission. With again all modes in PC1 and now both the slow propagating mode and the weakly evanescent mode in PC2, the transmission is close to perfect. This confirms that

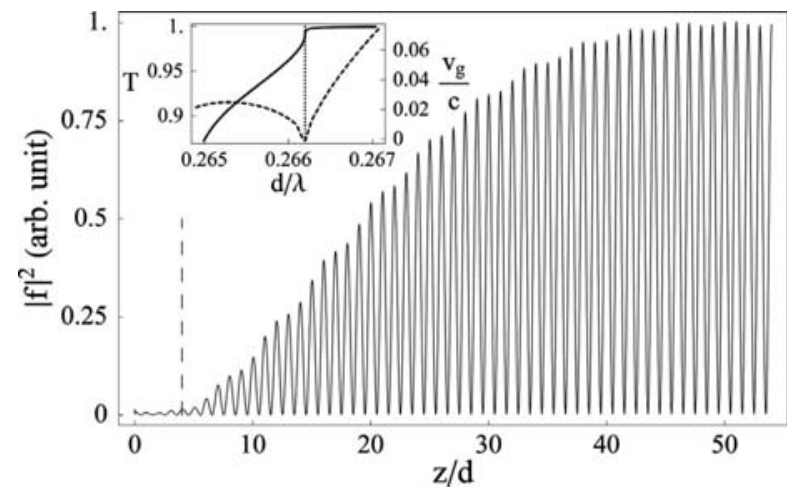

Fig. 4. Square modulus of the electric field when the light efficiently couples into the slow waveguide from the left. The vertical dashed line indicates the interface between PC1 and PC2. The inset shows the transmittance (solid curve) and the group velocity (dashed curve) versus frequency. the high transmission is associated with the inclusion of the weakly evanescent mode in PC2. The inclusion of other evanescent modes, for example the decaying mode with $\operatorname{Re}(k)<0$ [9] and no others, leads to inconsistent results, indicating that not enough modes are included to represent the fields satisfactorily.

While the result here is a "hero result" in that light is coupled into a mode with very high group index $\left(n_{g}>1000\right)$, other calculations, not given here, show that our result is quite generic. High coupling efficiency is achieved for modest values $n_{g} \approx 100$ as well and also essentially in any geometry in which the slow mode is associated with an inflection point, irrespective of how the band structure is engineered. For lower group indices, the evanescent mode also decays faster in PC2.

Thus efficient coupling into a slow mode is possible without transition region or other precaution. The reason this is possible, and the reason that the usual argument apparently showing the opposite is incomplete, is as follows: though slow modes have strong associated fields, this leads only to the conclusion that coupling is weak if evanescent modes are disregarded. When such modes are included, almost perfect coupling can be achieved. While we considered a two-dimensional geometry, our arguments were very general. Indeed, preliminary results from $3 \mathrm{D}$ finitedifference time domain simulations suggest that similar behavior occurs in realistic membrane PC geometries.

This work was produced with the assistance of the Australian Research Council under its ARC Centres of Excellence Program. T. P. White is supported by the EU-FP6 Marie Curie Fellowship SLIPPRY.

\section{References and Note}

1. Y. A. Vlasov, M. O'Booyle, H. F. Hamann, and S. J. McNab, Nature 438, 65 (2005).

2. T. D. Happ, M. Kamp, and A. Forchel, Opt. Lett. 26, 1102 (2001).

3. P. Velha, J. P. Hugonin, and P. Lalanne, Opt. Express 15, 6102 (2007).

4. C. M. de Sterke, J. Walker, K. B. Dossou, and L. C. Botten, Opt. Express 15, 10984 (2007).

5. J. P. Hugonin, P. Lalanne, T. P. White, and T. F. Krauss, Opt. Lett. 32, 2638 (2007).

6. L. C. Botten, T. P. White, A. A. Asatryan, T. N. Langtry, C. M. de Sterke, and R. C. McPhedran, Phys. Rev. E 70, 056606 (2004).

7. J. Ballato, A. Ballato, A. Figotin, and I. Vitebskiy, Phys. Rev. E 71, 036612 (2005).

8. D. Marcuse, Theory of Dielectric Optical Waveguides, 2nd ed. (Academic, 1991).

9. At negative $k[\operatorname{Re}(k) d /(2 \pi)=-0.38]$ there are also two evanescent modes, one of which decays with $z$. It has a field in which the $f_{ \pm}$are switched, and it therefore does not match the forward-propagating slow mode well; we therefore ignore it in our qualitative description, and indeed it plays virtually no role in the coupling. 